\title{
The association between neutrophil- lymphocyte ratio and poor outcomes following infant cardiac surgery
}

\author{
Peng Gao, Jinping Liu*, Xu Wang, Peiyao Zhang, Yu Jin, Liting Bai and Yixuan Li
}

\begin{abstract}
Background: Neutrophil-lymphocyte ratio (NLR) is a valuable indicator for evaluating inflammation and adverse outcomes after cardiac surgery. The objective of this study was to evaluate the association of perioperative NLR with clinical outcomes in infants undergoing congenital heart surgery with cardiopulmonary bypass.

Methods: We performed a retrospective review of 424 consecutive infants ( $\leq 1$ year) undergoing cardiac surgery between January 2019 and September 2019. Neonates ( $\leq 28$ days) and patients with incomplete NLR data were excluded. The study endpoint was a composite of poor outcomes after surgery. We assess the correlation between perioperative NLR and clinical outcomes. A receiver operating characteristic curve and multivariable logistic regression were applied to identify the prognosis performance of postoperative NLR for poor outcomes.
\end{abstract}

Results: A total of 68 (16\%) infants experienced at least one of the poor outcomes. Postoperative NLR on the third day after the surgery showed the best prognostic significance (AUC $=0.763,95 \% \mathrm{Cl} 0.700-0.826$ ) among perioperative period, with a cut-off value of 2.05. Postoperative NLR was also strongly correlated with mechanical ventilation time, length of ICU and hospital stay $(p<0.001)$. Multivariable logistic regression revealed that elevated postoperative NLR (OR 3.722, 95\%Cl 1.895-7.309, $p<0.001$ ) was an independent risk factor for poor outcomes in infants after cardiac surgery.

Conclusions: Postoperative NLR was correlated with increased mechanical ventilation time, length of ICU and hospital stay. Elevated postoperative NLR was an independent predictor for poor outcomes after cardiac surgery in infants.

Keywords: Pediatric cardiac surgery, Infants, Neutrophil-lymphocyte ratio, Inflammatory response, Poor outcomes

\section{Introduction}

Cardiopulmonary bypass (CPB) initiates systemic inflammatory response syndrome (SIRS), which is associated with adverse outcomes in pediatric cardiac surgery $[1$, 2]. However, few inflammatory biomarkers could be conveniently used to evaluate outcomes in current clinical practice. Neutrophil-lymphocyte ratio (NLR) has been

\footnotetext{
*Correspondence: liujinping@fuwai.com

Pediatric Cardiac Surgery Center, National Center for Cardiovascular Diseases, Fuwai Hospital, Chinese Academy of MedicalSciences and Peking Union Medical College, No. 167, North Lishi Road, Xicheng District 100037, China
}

reported as a useful marker of system inflammation in sepsis, chronic obstructive pulmonary disease, and abdominal operations [3-5], as a simple and readily available biomarker that was calculated from blood routine examination. Moreover, increased perioperative NLR was shown to be associated with poor outcomes after cardiac surgery in adult patients [6]. Recent studies showed that the higher preoperative NLR in children undergoing cardiac surgery was not only an independent risk factor for low cardiac output in the early postoperative period but also correlated with short-term and long-term mortality $[7,8]$. Besides, postoperative NLR in pediatric patients 
with cardiopulmonary bypass was significantly higher than those before the operation, which was consistent with increased duration of mechanical ventilation and ICU stay [9]. Although NLR is a valuable indicator for evaluating inflammation and clinical outcomes, limited evidence in the population of infants has been shown. We hypothesized that elevated NLR is relevant to poor outcomes after pediatric cardiac surgery. The objective of the present study was to evaluate the association of perioperative NLR with clinical outcomes in infants, thereby providing an underlying predictor for clinical prognosis.

\section{Patients and methods}

\section{Patients}

This is a retrospective observational study conducted at Fuwai Hospital (Beijing, China). From January 2019 to September 2019, a total of 497 infants ( $\leq 1$ year) undergoing cardiac surgery with $\mathrm{CPB}$ were consecutively included. Excluded were patients utilizing preoperative extracorporeal life support, without cardioplegic arrest, emergent surgery, or incomplete data of perioperative NLR. Neonates ( $\leq 28$ days) were also excluded because they have a special pathophysiological condition. The study was approved by Fuwai hospital and was conducted in accordance with the principles of the Declaration of Helsinki.

\section{Definitions}

For each pediatric patient, the data were retrospectively collected through the electronic database of the hospital information system. Peripheral venous blood was taken and sent to the laboratory for blood routine examination at the admission in pediatric intensive care unit (PICU) and every morning during PICU stay. NLR is the quotient of the absolute value of neutrophils and lymphocytes from the same blood routine examination. NLR on one day before the surgery was defined as Baseline NLR, the operation day as Day 0 and 1, 2, 3, 6 days after surgery respectively named as Day1, Day2, Day3, Day6.

Poor outcomes were limited to the postoperative duration of hospitalization, defined as patients undergoing at least one of death, pulmonary or incision infection, need for peritoneal dialysis, postoperative re-intubation or reoperation, the requirement of extracorporeal membrane oxygenation (ECMO), prolonged ICU stay $\geq 11$ days (90th percentile) and prolonged mechanical ventilation $\geq 100 \mathrm{~h}$ (90th percentile).

\section{Statistical analyses}

Categorical variables were presented as frequencies with percentages, continuous variables as mean \pm standard deviation, or median with interquartile ranges (IQR) $25-75$ th percentile. The normality of distribution was tested with the Shapiro-Wilk test. Chi-square or Fisher's exact test was used for categorical variables, and the t-test or Mann-Whitney $U$ test for continuous variables. As for the relationship between poor outcomes and NLR on different days, cut-off values were identified using a receiver operating characteristic (ROC) curve, and the area under the curve (AUC) was used to determine the accuracy of the diagnostic prediction. Select the NLR with the best diagnostic performance as the postoperative NLR. We performed a Spearman rank correlation test $(r)$ to assess the unadjusted relationships between preoperative and postoperative NLR with clinical outcomes (mechanical ventilation time, length of ICU and hospital stay).

To evaluate if postoperative NLR predicts poor outcomes, we built multivariable logistic regression modeling, adjusting for factors that were evaluable in the prediction of clinical outcome of pediatric heart surgery: age, gender, weight, Risk Adjustment for Congenital Heart Surgery (RACHS) score, and CPB duration [10]. The NLR model added postoperative NLR to the basic model included age, gender, weight, RACHS score, and $\mathrm{CPB}$ duration. ROC comparisons were performed between models to check discriminative ability. For each cut-off value, the sensitivity and specificity were measured.

For all the statistical tests, a $p$-value $<0.05$ was considered significant. The data were analyzed using statistical software SPSS 25.0 (SPSS Inc., Chicago, IL, USA) and R 4.1.0 (Institute for Statistics and Mathematics, Vienna, Austria).

\section{Results}

\section{Patient characteristics}

During the study period (January 2019 to September 2019), 497 pediatric patients were consecutively included. Due to incomplete NLR data, only 424 infants (219 male and 205 female) were included in the final analysis. The median age and weight was $6.74 \pm 2.83$ months and $6.81 \pm 1.55 \mathrm{~kg}$. The types of congenital heart disease were as follows: Ventricular septal defect 181 (42.7\%), Atrioventricular septal defect 41 (9.7\%), Atrial septal defect 36 (8.5\%), Tetralogy of Fallot 68 (16.0\%), Total anomalous pulmonary venous connection 15 (3.5\%), Double outlet right ventricle $14(3.3 \%)$, Coarctation of the aorta 14 (3.3\%), Pulmonary stenosis $11(2.6 \%)$, Transposition of the great arteries $9(2.1 \%)$, Total endocardial cushion defect 8 (1.9\%), interrupted aortic arch 6 (1.4\%), Pulmonary atresia $5(1.2 \%)$, and other complex diagnoses 16 (3.8\%). The number of patients with poor outcomes was 68/424 (16.0\%). Results was presented in Table 1. 
Table 1 Characteristics of the study population

\begin{tabular}{ll}
\hline Variables & $\mathbf{N}=\mathbf{4 2 4}$ \\
\hline Age (months) & $6.74 \pm 2.83$ \\
Male & $219(51.7 \%)$ \\
Weight (kg) & $6.81 \pm 1.55$ \\
RACHS-1 score $\geq 3$ & $146(34.4 \%)$ \\
CPB duration (min) & $90.59 \pm 41.28$ \\
ACC duration (min) & $58.59 \pm 31.20$ \\
Poor outcomes & $68(16.0 \%)$ \\
Type of CHD & \\
Ventricular septal defect & $181(42.7 \%)$ \\
Atrioventricular septal defect & $41(9.7 \%)$ \\
Atrial septal defect & $36(8.5 \%)$ \\
Tetralogy of Fallot & $68(16.0 \%)$ \\
Total anomalous pulmonary venous connection & $15(3.5 \%)$ \\
Double outlet right ventricle & $14(3.3 \%)$ \\
Coarctation of the aorta & $14(3.3 \%)$ \\
Pulmonary stenosis & $11(2.6 \%)$ \\
Transposition of the great arteries & $9(2.1 \%)$ \\
Total endocardial cushion defect & $8(1.9 \%)$ \\
Interrupted aortic arch & $6(1.4 \%)$ \\
Pulmonary atresia & $5(1.2 \%)$ \\
Other complex diagnoses & $16(3.8 \%)$ \\
\hline RACHS-1, Risk Adjustment for Congenta Heat Surger-1;CPB &
\end{tabular}

RACHS-1, Risk Adjustment for Congenital Heart Surgery-1; CPB, cardiopulmonary bypass; $\mathrm{ACC}$, aorta cross-clamping; $\mathrm{CHD}$, congenital heart surgery

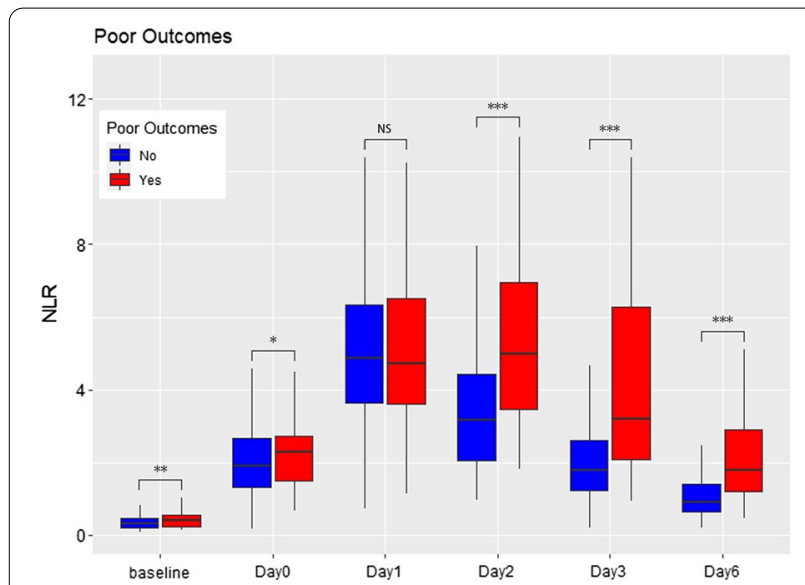

Fig. 1 Perioperative NLR variation in infants with poor outcomes and non-poor outcomes (* indicates a $p$-value at $0.05-0.01, * *$ indicates a $p$-value at $0.01-0.001,{ }^{* * *}$ indicates a $p$-value $<0.001$ )

\section{Perioperative NLR and clinical outcomes}

NLR variation among different days between infants with poor outcomes and non-poor outcomes is illustrated in Fig. 1. A definite trend is evident that perioperative NLR increased from baseline due to the
Table 2 ROC comparison of NLR on different days for predicting poor outcomes

\begin{tabular}{lrrrl}
\hline Variables & $\boldsymbol{p}$ value & AUC & $\mathbf{9 5 \% C l}$ & Cut-off Value \\
\hline Baseline NLR & 0.018 & 0.591 & $0.516-0.666$ & 0.37 \\
Day 0 NLR & 0.098 & 0.563 & $0.491-0.636$ & - \\
Day 1 NLR & 0.717 & 0.514 & $0.436-0.592$ & - \\
Day 2 NLR & $<0.001$ & 0.719 & $0.658-0.781$ & 5.24 \\
Day 3 NLR & $<0.001$ & 0.763 & $0.700-0.826$ & 2.05 \\
Day6 NLR & $<0.001$ & 0.748 & $0.679-0.817$ & 1.38 \\
\hline
\end{tabular}

ROC, receiver operating characteristic; NLR, neutrophil-lymphocyte ratio

Table 3 Correlation between perioperative NLR and clinical outcomes

\begin{tabular}{llllll}
\hline Variables & \multicolumn{2}{l}{ Preoperative NLR } & & \multicolumn{2}{l}{ Postoperative NLR } \\
\cline { 2 - 3 } & Coefficient & $\boldsymbol{p}$-value & & Coefficient & $\boldsymbol{p}$ value \\
\hline MV time & 0.148 & 0.002 & & 0.554 & $<0.001$ \\
ICU LOS & 0.110 & 0.023 & & 0.469 & $<0.001$ \\
Hospital LOS & 0.081 & 0.094 & & 0.421 & $<0.001$ \\
\hline
\end{tabular}

$\mathrm{NLR}$, neutrophil-lymphocyte ratio; MV, mechanical ventilation; ICU, intensive care unit; LOS, length of stay

operation and CPB. NLR in patients without poor outcomes increased to a peak one day after the surgery and then decreased gradually. However, postoperative NLR in patients with poor outcomes maintained at a high level within 3 days after the surgery and still higher until Day6.

Baseline, Day0, Day2, Day3, and Day6 NLR showed statistical significance between the two groups. Repeated measurement analysis of variance showed that NLR was different in two groups, including both single NLR $(F=330.529, p<0.001)$, an interaction term between NLR and poor outcomes $(F=19.416$, $p<0.001)$. To explore the predictive value of NLR on different days, ROC curve analysis with NLR and poor outcomes was applied, results are shown in Table 2. According to AUC and $p$-value, Baseline, Day2, Day3, and Day6 NLR have a predictive diagnostic effect, with cutoff values of $0.37,5.242 .05$, and 1.38 respectively, and Day3 NLR (AUC $=0.763,95 \%$ CI $0.700-0.826$ ) showed the best prognostic significance (Table 2).

Since Day3 NLR showed a larger AUC than other days, we choose it as postoperative NLR in the following analysis. As presented in Table 3, preoperative NLR was significantly correlated with mechanical ventilation time $(p=0.002)$ and ICU length of stay $(p=0.023)$ but had no obvious correlation with hospital length of stay $(p=0.081)$. Postoperative NLR was closely related to mechanical ventilation time $(p<0.001)$, length of ICU $(p<0.001)$ and hospital stay $(p<0.001)$. 


\section{Characteristics and poor outcomes of patients with elevated postoperative NLR}

According to postoperative NLR cutoff thresholds (2.05), 424 infants were grouped to evaluate the differences in characteristics and poor outcomes of patients with elevated postoperative NLR. As presented in Table 4, patients with elevated postoperative NLR were younger and lighter in weight. They underwent more complex surgery (RACHS-1 $\geq 3$ ), repeated $\mathrm{CPB}$ and circulatory arrest, as well as a longer duration of $\mathrm{CPB}$ and aorta cross-clamping (ACC). Meanwhile, cyanotic heart disease and pulmonary arterial hypertension were more common compared with patients with non-elevated postoperative NLR.

Among the study population, 54/195 (27.7\%) of elevated postoperative NLR $(\geq 2.05)$ patients experienced at least one of the poor outcomes compared to only $6.1 \%$ $(14 / 229)$ without elevated postoperative NLR $(p<0.001)$. Infants in the elevated NLR group had a higher incidence of comorbidity: postoperative re-intubation $(9.2 \%$ vs $1.3 \%, p<0.001)$, re-operation $(7.7 \%$ vs $1.7 \%, p=0.003)$, need for peritoneal dialysis ( $3.6 \%$ vs $0 \%, p=0.004)$, pulmonary infection $(25.1 \%$ vs $8.1 \%, p<0.001)$, prolonged ICU stay $(20.5 \%$ vs $3.5 \%, p<0.001)$, and prolonged mechanical ventilation time $(20.5 \%$ vs $3.9 \%, p<0.001)$. However, differences in death $(1.5 \%$ vs $0.9 \%, p=0.665)$, incision infection ( $0 \%$ vs $0.9 \%, p=0.502)$ and requirement of ECMO $(1.0 \%$ vs $0 \%, p=0.211)$ were not significant between the two groups. Median mechanical ventilation time, length of ICU and hospital stay were

Table 4 Comparison of characteristics between the elevated postoperative NLR and non-elevated postoperative NLR patients

\begin{tabular}{|c|c|c|c|}
\hline Variables & $\begin{array}{l}\text { NLR }<2.05 \\
(n=229)\end{array}$ & $\begin{array}{l}\text { NLR } \geq 2.05 \\
(n=195)\end{array}$ & $p$ value \\
\hline Age (month) & $7.18 \pm 2.62$ & $6.23 \pm 2.98$ & 0.001 \\
\hline Male & $116(50.7 \%)$ & $103(52.8 \%)$ & 0.675 \\
\hline Weight (kg) & $7.02 \pm 1.55$ & $6.56 \pm 1.53$ & 0.002 \\
\hline RACHS-1 score $\geq 3$ & $65(28.4 \%)$ & $81(41.5 \%)$ & 0.004 \\
\hline Preoperative LVEF (\%) & $67.46 \pm 5.54$ & $66.31 \pm 7.78$ & 0.087 \\
\hline $\begin{array}{l}\text { Pulmonary arterial hyperten- } \\
\text { sion }\end{array}$ & $92(40.2 \%)$ & $105(53.8 \%)$ & 0.005 \\
\hline Cyanotic heart disease & $58(25.3 \%)$ & $81(41.5 \%)$ & $<0.001$ \\
\hline CPB duration (min) & $76.29 \pm 28.22$ & $107.37 \pm 47.50$ & $<0.001$ \\
\hline ACC duration (min) & $48.44 \pm 21.50$ & $70.51 \pm 36.25$ & $<0.001$ \\
\hline Repeated CPB & $3(1.3 \%)$ & $10(5.2 \%)$ & 0.022 \\
\hline Lower body circulatory arrest & $3(1.3 \%)$ & $8(4.1 \%)$ & 0.069 \\
\hline $\begin{array}{l}\text { Lowest temperature during } \\
\text { CPB }\left({ }^{\circ} \mathrm{C}\right)\end{array}$ & $31.0 \pm 1.31$ & $30.01 \pm 2.12$ & $<0.001$ \\
\hline Lowest Hct during CPB (\%) & $23.68 \pm 2.77$ & $23.92 \pm 3.12$ & 0.401 \\
\hline
\end{tabular}

RACHS, Risk Adjustment for Congenital Heart Surgery; LVEF, left ventricular ejection fraction; $\mathrm{CPB}$, cardiopulmonary bypass; $\mathrm{ACC}$, aorta cross-clamping
26 h, 5 days and 17 days in elevated NLR patients, respectively, but only 9 h, 3 days, and 12 days in non-elevated NLR patients $(p<0.001)$. Results are shown in Table 5.

\section{The predictive effect of $\mathrm{nlr}$ on poor outcomes}

As presented in Table 6, the multivariable logistic regression model showed that postoperative NLR (OR 1.298, 95\%CI 1.153-1.461, $p<0.001)$ predicted a composite of poor outcomes, after adjusting for basic clinical predictions (age, gender, weight, RACHS score, and CPB

Table 5 Comparison of poor outcomes between the elevated postoperative NLR and non-elevated postoperative NLR patients

\begin{tabular}{lllr}
\hline Variables & $\begin{array}{l}\text { NLR }<2.05 \\
(\mathbf{n}=\mathbf{2 2 9})\end{array}$ & $\begin{array}{l}\text { NLR } \geq \mathbf{2 . 0 5} \\
(\mathbf{n}=\mathbf{1 9 5})\end{array}$ & $\boldsymbol{p}$ value \\
\hline Poor outcomes & $14(6.1 \%)$ & $54(27.7 \%)$ & $<0.001$ \\
Re-intubation & $3(1.3 \%)$ & $18(9.2 \%)$ & $<0.001$ \\
Death & $2(0.9 \%)$ & $3(1.5 \%)$ & 0.665 \\
Re-operation & $4(1.7 \%)$ & $15(7.7 \%)$ & 0.003 \\
ECMO & 0 & $2(1.0 \%)$ & 0.211 \\
Peritoneal dialysis & 0 & $7(3.6 \%)$ & 0.004 \\
Incision infection & $2(0.9 \%)$ & 0 & 0.502 \\
Pulmonary infection & $19(8.3 \%)$ & $49(25.1 \%)$ & $<0.001$ \\
Prolonged MV time & $9(3.9 \%)$ & $40(20.5 \%)$ & $<0.001$ \\
Prolonged ICU LOS & $8(3.5 \%)$ & $40(20.5 \%)$ & $<0.001$ \\
MV time & $9(6,19)$ & $26(18,86)$ & $<0.001$ \\
ICU LOS & $3(1,4)$ & $5(3,8)$ & $<0.001$ \\
Hospital LOS & $12(10,16)$ & $17(13,24)$ & $<0.001$
\end{tabular}

NLR, neutrophil-lymphocyte ratio; ECMO, extracorporeal membrane oxygenation; MV, mechanical ventilation; ICU, intensive care unit; LOS, length of stay

Table 6 Multivariate logistic regression of poor outcomes

\begin{tabular}{lrrrl}
\hline Variables & Wald & $\boldsymbol{p}$-value & OR & $\mathbf{9 5 \%} \mathbf{C l}$ \\
\hline NLR model 1 & & & & \\
Gender & 5.185 & 0.023 & 2.062 & $1.106-3.844$ \\
Weight & 9.964 & 0.002 & 0.716 & $0.581-0.881$ \\
CPB duration & 13.025 & $<0.001$ & 1.016 & $1.006-1.021$ \\
Postoperative NLR & 18.620 & $<0.001$ & 1.298 & $1.153-1.461$ \\
NLR model 2 & & & & \\
Gender & 8.662 & 0.003 & 2488 & $1.356-4.565$ \\
Weight & 11.378 & 0.001 & 0.708 & $0.580-0.865$ \\
CPB duration & 13.816 & $<0.001$ & 1.012 & $1.006-1.019$ \\
Elevated postoperative NLR & 14.561 & $<0.001$ & 3.722 & $1.895-7.309$ \\
NLR model 3 & & & & \\
Gender & 4.353 & 0.037 & 1.861 & $1.038-3.334$ \\
Age & 9.960 & 0.002 & 0.849 & $0.767-0.940$ \\
CPB duration & 27.261 & $<0.001$ & 1.017 & $1.011-1.023$ \\
Preoperative NLR & 2.759 & 0.097 & 1.739 & $0.905-3.341$ \\
\hline
\end{tabular}

$\mathrm{NLR}$, neutrophil-lymphocyte ratio; CPB, cardiopulmonary bypass; 
duration). When NLR was analyzed as a categorical variable, elevated postoperative NLR $(\geq 2.05)$ (OR 3.722, 95\%CI 1.895-7.309, $p<0.001)$ was also an independent risk factor for poor outcomes. However, preoperative NLR showed no prognostic significance for poor outcomes $(p=0.097)$.

The basic model included age, gender, weight, RACHS score, and $\mathrm{CPB}$ duration demonstrated good predictive power (AUC $0.759,95 \% \mathrm{CI} 0.693-0.826$ ), with better discrimination in the NLR Model 1 (AUC 0.813, 95\%CI 0.754-0.872) and NLR Model 2 (AUC 0.797, 95\%CI 0.739-0.855) containing postoperative NLR as a categorical variable. Results are shown in Table 7 . The NLR Model 1 and NLR Model 2 demonstrated improvement on the basic model as measured by the absolute difference in AUC, respectively $(\triangle \mathrm{AUC}=0.0538, p=0.0043)$ and $(\triangle \mathrm{AUC}=0.0378, p=0.0436)$. The NLR Model $1 \mathrm{had}$ the best predictive performance among the three models (sensitivity $82.35 \%$, specificity $71.63 \%$ ). ROC comparison between the NLR model and basic model is illustrated in Fig. 2

\section{Comment}

The main findings of this study were that postoperative NLR showed an obvious increase as a result of cardiac surgery and CPB. Postoperative NLR was associated with poor outcomes in infants undergoing congenital heart surgery, and NLR on the third day after surgery had the best predictive performance with a cut-off value of 2.05 . After adjustment for other risk factors, the elevated postoperative NLR still emerged as an independent predictor of poor outcomes. In addition, postoperative NLR was significantly correlated with mechanical ventilation time, length of ICU and hospital stay. The result suggests that postoperative NLR could be a predictor for poor outcomes in infants undergoing $\mathrm{CPB}$.

Systemic inflammatory response syndrome is frequently observed after cardiac surgery, which plays an important role in the pathogenesis of adverse clinical outcomes related to cardiopulmonary bypass and surgical trauma $[11,12]$. The comprehensive contact of blood with the surface of the cardiopulmonary bypass circuit causes an inflammatory cascade of body fluid (pro-inflammatory cytokines, complement, and blood

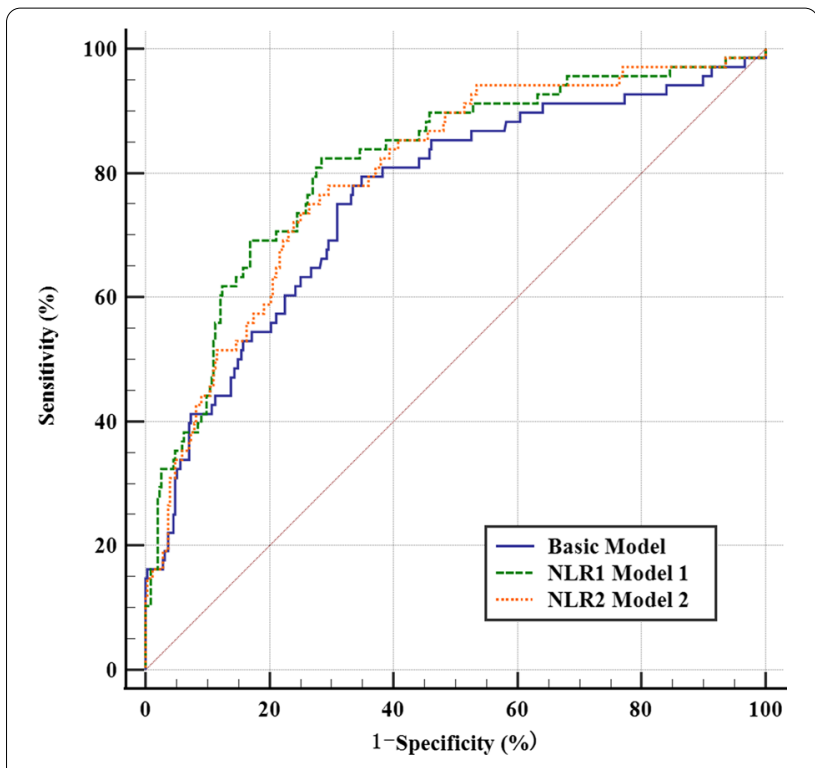

Fig. 2 ROC comparison between the NLR model and basic model

coagulation system), as well as cells (leukocytes, platelets, and vascular endothelial cells) [13]. These inflammatory pathways are complicated and interconnected in multiple ways with each other, exaggerating their mutual effect. Meanwhile, ischemia-reperfusion injury and endotoxemia, as a result of hypothermic advection perfusion or even circulatory arrest, leads to vascular endothelial injury by release of reactive oxygen species and variations of the microcirculation [2]. Compared with adult patients, the inflammatory response is more severe in pediatric patients undergoing CPB [14]. Many studies have indicated a higher incidence of SIRS in children with younger age or lower body weight after CPB $[15,16]$. Due to the lower body weight, the pediatric patient's total blood volume is seriously mismatched with the surface area of the cardiopulmonary bypass circuit and priming volume, leading to greater activation of the inflammatory response and theoretically higher risk of SIRS [17]. What's more, younger children may experience a greater risk of SIRS for their immature organ function and a higher metabolic rate $[1,17]$.

Table 7 ROC comparison of different models for predicting poor outcomes

\begin{tabular}{|c|c|c|c|c|c|}
\hline Variables & AUC $(95 \% \mathrm{Cl})$ & Sensitivity & Specificity & Youden index & $p$-value \\
\hline Basic model & $0.759(0.693-0.826)$ & 79.41 & 65.17 & 0.4458 & $<0.001$ \\
\hline NLR model 1 & $0.813(0.754-0.872)$ & 82.35 & 71.63 & 0.5398 & $<0.001$ \\
\hline NLR model 2 & 0.797 (0.739-0.855) & 75.00 & 73.60 & 0.4860 & $<0.001$ \\
\hline
\end{tabular}

ROC, receiver operating characteristic; NLR, neutrophil-lymphocyte ratio 
Although a variety of biomarkers of inflammatory response have been determined in the prediction and diagnosis of adverse outcomes, few could readily and conveniently use in current clinical practice. NLR has been paid more attention for its significant features of low price, immediate access, direct and repeatable detection. Various evidence regards NLR as a novel marker, which can reflect the level of systematic inflammation $[18,19]$ and a good indicator for predicting and diagnosing prognostic outcomes in several diseases [20-22]. The neutrophil is an essential component of the systemic inflammatory response to tissue injury and reperfusion injury [23]. Converted WBC subtypes, specifically elevated neutrophils with reduced lymphocytes represent an inflammatory process that has been associated with increased risk of death and myocardial infarction in patients with coronary artery disease [24]. Recent studies indicated NLR was an independent predictor of adverse outcomes and harmed long-term survival after openheart surgery in adult patients [25].

In adult patients, an increase in perioperative NLR was associated with raised mortality and morbidity in patients undergoing cardiac surgery [26], which may guide perioperative management and risk-stratification of patients. Many investigators have also proposed and proved the application of NLR in pediatric heart surgery. $\mathrm{Xu}$ et al. [9] found that postoperative NLR increased significantly after open-heart surgery with cardiopulmonary bypass, which was consistent with prolonged mechanical ventilation time and ICU length of stay. Manuel et al. [27] retrospectively analyzed 141 univentricular patients, results showed preoperative NLR $>2$ was associated with an increase in prolonged total hospital length of stay, duration of mechanical ventilation, and ICU length of stay, and these pediatric patients had higher mortality in 24 months after the operation. Moreover, a recent study [28] also reported NLR could predict prolonged mechanical ventilation as a novel indicator, which was closely related to early postoperative clinical outcomes. Savluk and colleagues [8] evaluated the impact of preoperative NLR in Norwood stage I operations. They demonstrated a correlation between high preoperative NLR with an increased risk of death in 53 patients. Besides, Iliopoulos et al. [7] indicated that elevated preoperative neutrophil-lymphocyte ratio had almost three times more risk to develop low cardiac output in children after congenital heart surgery.

In the current study, preoperative NLR was not an independent risk factor for predicting postoperative adverse outcomes, which was different from the conclusions of previous studies $[8,27,29]$. Although preoperative NLR was associated with mechanical ventilation time and length of ICU stay like other studies, the small coefficient indicated a weak correlation. Meanwhile, postoperative NLR on the first day after surgery showed no prognostic significance. The disparate results limit the application of NLR in predicting clinical outcomes during early postoperative period in infants after $\mathrm{CPB}$. Most subjects in previous studies were older than infants and the endpoints varied from each other, which may help explain the difference in results. In addition, NLR on different days in pediatric heart surgery was not shown before. By comparing NLR on different perioperative days, we found that the NLR on the third day after surgery has the best predictive performance for poor outcomes, which was an independent risk factor at the same time. Furthermore, it has a strong correlation with mechanical ventilation time, length of ICU and hospital stay.

Our data exhibited that perioperative NLR rose to a peak on the first day after surgery in infants undergoing cardiac surgery. Compared with the infants without poor outcomes, whose NLR gradually decreased after surgery, patients with poor outcomes maintained a high level of postoperative NLR for a week or even longer, which indicates a sustained high level of inflammatory response. This might be the potential performance of the poor outcomes rather than the cause, however, postoperative NLR did have a significant correlation with poor prognosis and clinical outcomes. Our results suggest the importance to pay attention to the inflammatory response in the pediatric population. Thus, we speculate that active prevention of systemic inflammatory response may protect immature organs from injury, furthermore reduce postoperative mortality and morbidity.

There are a few limitations to our study. Firstly, as a retrospective study conducted in our hospital, it would inevitably be affected by systematic errors and bias. Secondly, 73 patients were excluded due to incomplete data, and most of them didn't have postoperative daily blood routine examination for their fast recovery from surgery, which may have a slight impact on the results. Finally, the specific relationship between NLR and inflammatory response remains unclear, because well-established inflammatory biomarkers such as inflammatory factors and C-reactive protein are not daily available. More further researches are needed before we fully understand this phenomenon.

\section{Conclusion}

This study demonstrated postoperative NLR was correlated with increased mechanical ventilation time, length of ICU and hospital stay. Elevated postoperative NLR $(\geq 2.05)$ was an independent predictor for poor outcomes following congenital heart surgery in infants. NLR was a good biomarker that showed satisfying prognostic significance and could be utilized as a convenient and low-cost 


\section{indicator for postoperative care after pediatric cardiac surgery.}

\author{
Abbreviations \\ NLR: Neutrophil-lymphocyte ratio; CPB: Cardiopulmonary bypass; SIRS: \\ Systemic inflammatory response syndrome; PICU: Pediatric intensive care unit; \\ RACHS: Risk adjustment for congenital heart surgery; ACC: Aorta cross-clamp- \\ ing; CHD: Congenital heart disease.
}

\section{Acknowledgements}

Not applicable.

\section{Authors' contributions}

Study design, data analysis, interpretation of results, drafting of manuscript GP. Interpretation of results, reviewing the manuscript:WX. Data collection, database design, reviewing the manuscript: ZPY, JY, BLT, LYX. Study design, interpretation of results, critical reviewing the manuscript: LJP. All authors read and approved the final manuscript.

\section{Funding}

This study was supported by CAMS Innovation Fund for Medical Sciences (No.2020-I2M-C\&T-B-063). The funders had no role in the study design, data collection and analysis, decision to publish, or preparation of the manuscript.

\section{Availability of data and materials}

The datasets used and analyzed during the current study are available from the corresponding author on reasonable request.

\section{Declarations}

\section{Ethics approval and consent to participate}

This study was approved by the Medical Ethics Committee of Fuwai Hospital, Peking Union Medical College and Chinese Academy of Medical Sciences (approval number: 2021-1483). Written informed consent was provided by all participants' legal guardians.

\section{Consent for publication}

Not applicable.

\section{Competing interests}

The authors declare no competing interests.

Received: 29 September 2021 Accepted: 26 October 2021 Published online: 08 November 2021

\section{References}

1. Brix-Christensen V. The systemic inflammatory response after cardiac surgery with cardiopulmonary bypass in children. Acta Anaesthesiol Scand. 2001:45(6):671-9.

2. Paparella D, Yau TM, Young E. Cardiopulmonary bypass induced inflammation: pathophysiology and treatment. An update. Eur J Cardiothorac Surg. 2002;21 (2):232-44.

3. Kong W, He Y, Bao H, Zhang W, Wang X. Diagnostic value of neutrophillymphocyte ratio for predicting the severity of acute pancreatitis: a metaanalysis. Dis Markers. 2020;2020:9731854.

4. Acartürk Tunçay E, Karakurt Z, Aksoy E, Saltürk C, Gungor S, Ciftaslan N, et al. Eosinophilic and non-eosinophilic COPD patients with chronic respiratory failure: neutrophil-to-lymphocyte ratio as an exacerbation marker. Int J Chron Obstruct Pulmon Dis. 2017;12:3361-70.

5. Da Silva M, Cleghorn MC, Elnahas A, Jackson TD, Okrainec A, Quereshy FA. Postoperative day one neutrophil-to-lymphocyte ratio as a predictor of 30-day outcomes in bariatric surgery patients. Surg Endosc. 2017;31(6):2645-50

6. Wang $Q, L i J$, Wang X. The neutrophil-lymphocyte ratio is associated with postoperative mortality of cardiac surgery. J Thorac Dis. 2021;13(1):67-75.
7. Iliopoulos I, Alder MN, Cooper DS, Villarreal EG, Loomba R, Sahay RD, et al. Pre-operative neutrophil-lymphocyte ratio predicts low cardiac output in children after cardiac surgery. Cardiol Young. 2020;30(4):521-5.

8. Savluk OF, Guzelmeric F, Yavuz Y, Ukil F, Yilmaz A, Cevirme D, et al. Neutrophil-lymphocyte ratio as a mortality predictor for Norwood stage I operations. Gen Thorac Cardiovasc Surg. 2019;67(8):669-76.

9. $\mathrm{Xu} \mathrm{H}$, Sun Y, Zhang $\mathrm{S}$. The relationship between neutrophil to lymphocyte ratio and clinical outcome in pediatric patients after cardiopulmonary bypass surgery: a retrospective study. Front Pediatr. 2019;7:308.

10. Brown KL, Ridout D, Pagel C, Wray J, Anderson D, Barron DJ, et al. Incidence and risk factors for important early morbidities associated with pediatric cardiac surgery in a UK population. J Thorac Cardiovasc Surg. 2019;158(4):1185-1196.e7.

11. Whitlock RP, Devereaux PJ, Teoh KH, Lamy A, Vincent J, Pogue J, et al. Methylprednisolone in patients undergoing cardiopulmonary bypass (SIRS): a randomised, double-blind, placebo-controlled trial. Lancet. 2015;386(10000):1243-53.

12. Lomivorotov V, Kornilov I, Boboshko V, Shmyrev V, Bondarenko I, Soynov I, et al. Effect of intraoperative dexamethasone on major complications and mortality among infants undergoing cardiac surgery: the DECISION randomized clinical trial. JAMA. 2020;323(24):2485-92.

13. Warren OJ, Smith AJ, Alexiou C, Rogers PL, Jawad N, Vincent C, et al. The inflammatory response to cardiopulmonary bypass: part 1-mechanisms of pathogenesis. J Cardiothorac Vasc Anesth. 2009;23(2):223-31.

14. Boehne M, Sasse M, Karch A, Dziuba F, Horke A, Kaussen T, et al. Systemic inflammatory response syndrome after pediatric congenital heart surgery: incidence, risk factors, and clinical outcome. J Card Surg. 2017;32(2):116-25.

15. Güvener M, Korun O, Demirtürk OS. Risk factors for systemic inflammatory response after congenital cardiac surgery. J Card Surg. 2015;30(1):92-6.

16. Pagowska-Klimek I, Świerzko AS, Michalski M, Głowacka E, Szala-Poździej A, Sokołowska A, et al. Activation of the lectin pathway of complement by cardiopulmonary bypass contributes to the development of systemic inflammatory response syndrome after paediatric cardiac surgery. Clin Exp Immunol. 2016;184(2):257-63.

17. Kozik DJ, Tweddell JS. Characterizing the inflammatory response to cardiopulmonary bypass in children. Ann Thorac Surg. 2006;81 (6):S2347-54.

18. Templeton AJ, McNamara MG, Šeruga B, Vera-Badillo FE, Aneja P, Ocaña A, et al. Prognostic role of neutrophil-to-lymphocyte ratio in solid tumors: a systematic review and meta-analysis. J Natl Cancer Inst. 2014;106(6):dju124

19. Russell CD, Parajuli A, Gale HJ, Bulteel NS, Schuetz P, de Jager CPC et al. The utility of peripheral blood leucocyte ratios as biomarkers in infectious diseases: a systematic review and meta-analysis. J Infect. 2019;78(5):339-48.

20. Christoforaki V, Zafeiriou Z, Daskalakis G, Katasos T, Siristatidis C. First trimester neutrophil to lymphocyte ratio (NLR) and pregnancy outcome. J Obstet Gynaecol. 2020;40(1):59-64.

21. Hirahara T, Arigami T, Yanagita S, Matsushita D, Uchikado Y, Kita Y, et al. Combined neutrophil-lymphocyte ratio and platelet-lymphocyte ratio predicts chemotherapy response and prognosis in patients with advanced gastric cancer. BMC Cancer. 2019;19(1):672.

22. Takamori S, Tagawa T, Toyokawa G, Shimokawa M, Kinoshita F, Kozuma $Y$, et al. Prognostic impact of postoperative skeletal muscle decrease in non-small cell lung cancer. Ann Thorac Surg. 2020;109(3):914-20.

23. Segel GB, Halterman MW, Lichtman MA. The paradox of the neutrophil's role in tissue injury. J Leukoc Biol. 2011;89(3):359-72. https://doi.org/10. 1189/jlb.0910538.

24. Horne BD, Anderson JL, John JM, Weaver A, Bair TL, Jensen KR, et al. Which white blood cell subtypes predict increased cardiovascular risk? J Am Coll Cardiol. 2005;45(10):1638-43.

25. Silberman S, Abu-Yunis U, Tauber R, Shavit L, Grenader T, Fink D, et al. Neutrophil-lymphocyte ratio: prognostic impact in heart surgery. Early outcomes and late survival. Ann Thorac Surg. 2018;105(2):581-6.

26. Tan TP, Arekapudi A, Metha J, Prasad A, Venkatraghavan L. Neutrophillymphocyte ratio as predictor of mortality and morbidity in cardiovascular surgery: a systematic review. ANZ J Surg. 2015;85(6):414-9.

27. Manuel V, Miana LA, Guerreiro GP, Tenório DF, Turquetto A, Penha JG, et al. Prognostic value of the preoperative neutrophil-lymphocyte ratio in patients undergoing the bidirectional Glenn procedure. J Card Surg. 2020:35(2):328-34. 
28. Yin X, Xin M, Ding S, Gao F, Wu F, Wang J, et al. Predictive role of perioperative neutrophil to lymphocyte ratio in pediatric congenital heart disease associated with pulmonary arterial hypertension. BMC Surg. 2021;21(1):3.

29. Manuel V, Miana LA, Turquetto A, Guerreiro GP, Fernandes N, Jatene MB.

The role of the neutrophil-lymphocyte ratio for pre-operative risk stratification of acute kidney injury after tetralogy of Fallot repair. Cardiol Young. 2021;31(6):1009-14

\section{Publisher's Note}

Springer Nature remains neutral with regard to jurisdictional claims in published maps and institutional affiliations.
Ready to submit your research? Choose BMC and benefit from:

- fast, convenient online submission

- thorough peer review by experienced researchers in your field

- rapid publication on acceptance

- support for research data, including large and complex data types

- gold Open Access which fosters wider collaboration and increased citations

- maximum visibility for your research: over 100M website views per year

At BMC, research is always in progress.

Learn more biomedcentral.com/submissions 